\title{
UN PROBLEMA SUBESTIMADO EN ENFERMERIA: LA IN- FLUENCIA QUE EJERCE SOBRE LA ATENCIÓN AL PACIENTE EL BIENESTAR ECONÓMICO Y SOCIAL DE LA ENFERMERA
}

\begin{tabular}{l|c|} 
& $\mathrm{RBEn} / 02$ \\
\hline
\end{tabular}

JACOX, A. - Un problema subestimado en enfermeria: la influencia que ejerce sobre la atención al paciente el bienestar económico y social de la enfermera. Rev. Bras. Enf.; DF, $32: 8-19$, 1579.

\section{INTRODUCCIÓN}

El principio de la acción colectiva es fundamental para la obtención de las metas profesionales. Con la creación de una asociación profesional, los profesionales ponen de manifiesto su convicción de que tanto la profesión como los servicios de sus miembros, pueden ser mejorados por medio de una acción común antes que por esfuerzos individuales.

Si bien desde hace largo tiempo las enfermeras actúan colectivamente en algunos campos como el establecimiento de disposiciones especiales para programas de estudio y conceción de diplomas, la acción en favor de su propio bienestar económico y social no ha sido, por el contrario, objeto de igual interés. Este descuido ha contribuído directamente a mantener condiciones de tra- bajos insatisíactorias, bajos salarios $\mathrm{y}$ escasas satisfacciones profesionales, todo lo cual ha creado una situación en que la escasez continua de enfermeras y la dificultad de atraer candidatas capaces y mantenerlas en la profesión se hace totalmente evidente. La falta de personal de enfermeria calificado y estable contribuye, a sua vez, a que la. atención al paciente sea en muchos casos insuficiente. Para comprender esta sucesión de acontecimientos pareceria útil examinar las tendencias históricas y sociales que han influenciado a las enfermeras y a la atención de enfermería. Consideraremos primero en este trabajo, las funciones que deben desempeñar una enfermera, como éstas funciones han sido tradicionalmente consideradas como trabajo femenino, y los resultados de esta posición en cuanto a la escasez de personal de enfermería.

\footnotetext{
* Enfermera, dostora en filosofia. Decana Asociada del Proşrama de Investigación y de Desarrollo de Estudios a nivel de Doctorado en Enfermeria - Colorado - Denver - Publicación autorizada del CIE - Consejo Internacional de Enfermeras.
} 
JACOX, A. - Un problema subestimado en enfermeria: la influencia que ejerce sobre la atención al paciente el bienestar económico y social de la enfermera. Rev. Bras. Enf.; DF, $32: 8-19,1679$.

Abordaremos luego el concepto de profesionalismo y cómo las enfermeras han atendido algunos aspectos de la profesión al mismo tiempo que ignoraban otros, lo cual ha sido perjudicial para la enfermeria. Finalmente presentaremos la noción de acción colectiva profesional como sugerencia de una posible actitud a ser asumida por las enfermeras.

\section{FUNCIONES DE LA ENFERMERA}

La profesión de enfermería tiene an. tiguas raíces históricas en todos los países del mundo. Aunque la organización de le enfermería difiere entre los países en aspectos como qué tareas desempeña la enfermera, quilén hace qué, nivel minimo de educación, etc., en lo que se refiere a las funciones básicas de la enfermera se advierte una uniformidad internacional. Esas funciones comprenden generalmente la promoción y la restauración de la salud, la prevención de la enfermedad y e? cuidado de los enfermos. Aún cuando algunas de éstas flunciones son responsabilidad también de otras profesiones de salud, son las enfermeras quienes han proporcionado los cuidados necesarios a los enfermos.

En este sentido los conocimientos propios de la enfermeria pueden ser considerados como la ciencia de los cuidaúos, así como la ciencia de la curación es propia de la medicina. La ciencia de la curación comprende el diagnóstico y el tratamiento de la enfermedad, dominio reconocido del médico. En cambio, la ciencia de los cuidados está enfocada hacia los conocimientos necesarios para la atención de los enfermos, y comprende el confort, el alivio del dolor y otras sensaciones desagradables, la creación de condiciones favorables para el reposo y el sueño, la minimización de las complicaciones creadas por la hospitaliza- ción, la ayuda al paciente para que pueda sobrellevar las consecuencias de su enfermedad, la enseñanza a enfermos y familiares de la medida en que se debe asumir la responsabilidad do algunos aspectos de la enfermedad y la atención necesaria.

Si bien algunas actividades de la enfermera se relacionan directamente con el cumplimiento del tratamiento ordenado por el médico y dirigido a la curación, y algunas tareas del médico comprenden aspectos del cuidado de los enfermos, en general los dos campos del cuidado y la curación son del dominio respectivo de la enfermera y del médico. Las funciones del cuidado y la curación son complementarias puesto que ambos son aspectos necesarios e importantes de la salud. Tanto a la enfermería como a la medicina interesan otros aspectos de la atención sanitaria como la promoción de la salud y la prevención de la enfermedad, pero son las funciones principales de la atención y de la curación las que son propias e enfermeras y médicos.

La enfermería, la medicina y otras profesiones sanitarias deben interesarsc, no solamente en mejorar sua propia práctica, sino además en mantener $y$ superar las normas sanitarias fijadas Ninguna profesión sanitaria puede operar aisladamente sin considerar el contexto total de la atención de la salud. Si bien cada profesión se concentra en principio en su propia práctica y sus propios profesionales, debe estar preparada a hacerse cargo si es necesario, do otros aspectos del contexto sanitario y social en que opera.

\section{LA ENFERMERIA COMO TRABAJO FEMENINO}

La enfermeria ha sido tradicionalmente considerada como un trabajo para mujeres, y el cuidado de los en- 
JACOX, A. - Un problema subestimado en enfermeria: la influencia que ejerce sobre la atención al paciente el bienestar económico y social de la enfermera. Rev. Bras. Enf.; DF, $32: 8-19,1979$.

fermos ha estado en general en manos de enfermeras, aunque la situción varie ligeramente de un país a otro. Son generalmente las mujeres de la familia quienes se ocupan de los enfermos de la casa $\mathrm{y}$, consecuentemente, son mujeres las primeras en haver sido preparadas para el cuidado de los enfermos cuando se necesitó un grado de conocimientos más elevado que el que posee una persona sin profesión o especialización. Además de esta identificación con el sexo femenino, la enfermeria ha estado también identificada como una vocación religiosa. A través de la historia de la religión organizada sabemos que una de las más importantes tareas de sus integrantes, especialmente de las mujeres, ha sido la de suministrar ayuda y confort a los enfermos. Esto era considerado como parte de sus obligaciones y por lo tanto no se esperaba que fuera económicamente remunerado. La identificaeión con mujeres y religiosas llevaba en sí misma una fuerte dosis de sacrificio y de obediencia. Se esperaba generalmente, que las enfermeras consideraran las necesidades de sus pacientes antes que las suyas propias en el aspecto económico y social, y que además obedecieran siempre a sus "supe.riores", considerándose como tales a los médicos y otras enfermeras de mayor autoridad. Estas continúan siendo características de las enfermeras de nuestros dias.

Por ser identificada como trabajo femenino, la importancia de la enfermería es a menudo subestimada, como lo son otras tareas que están a cargo de mujeres: el cuidado de los niños, la cocina, la limpieza y el cuidado del hoga. y la familia en general. Contribuye a la devaluación de las funciones de la enfermería el hecho de que muchos aspectos del cuidado de los enfermos requieren, para su desempeño, habilidades o técnicas de tipo manual. En mu- chos grupos humanos las personas que trabajan con sus manos son consideradas como inferiores a las que trabajan con su cabeza. Aún cuando las enfermeras necesitan para desempeñarse tanto unas como la otra, la inclusión de tareas manuales entre sus obligaciones ha determinado la deteriorización de la imagen de la profesión. La identificación histórica de la enfermería como profesión de mujeres y como vocación religiosa, junto con el importante elemento que es el trabajo manual en enfermeria, ha ejercido una decisiva influencia en la manera de la cual la enfermeria ha evolucionado y es socialmente considerada.

\section{CONSECUENCIAS DE LA ESCASEZ DE ENFERMERAS}

La combinación de arduo trabajo y enfasis en el sacrificio y la obediencia ha hecho que la enfermeria atraiga en cuanto a vocación religiosa, pero no para que se la considere en general, como una ocupación atractiva. La escasez de enfermeras competentes es un problema al cual se enfrentan hoy en día casi todos los países del mundo. E] problema surge en cuanto al reclutamiento de candidatas y también que dejen la profesión.. A ello se agrega el hecho de que las enfermeras que están preparadas para un servicio profesion:t de primera categoría no tienen, en $1: 1$ mayoría de los casos, tiempo suficiente para dedicar al cuidado directo del paciente, y deben por el contrario dedicarse a enseñar y supervisar personas con menor preparación o a coordinar las actividades de otros profesionales de ln salud. Es un hecho reconocido desde hace tiempo, que las enfermeras mejor preparadas y más eficaces en la atención de enfermos son las primeras en ser promovidas a otro tipo de tareas. De esta manera los pacientes quedan 
JACOX, A. - Un problema subestimado en enfermeria: la influencia que ejerce sobre la atención al paciente el bienestar económico y social de la enfermera. Rev. Bras. Enf.; DF, $32: 8-19,1579$.

en manos de personas que poseen una formación incompleta o improvisada.

Otra consecuencia negativa de la escasez de enfermeras calificadas es que, aquellas que lo son, no cuentan con tiempo suficiente para dedicar al mejoramiento del nivel general de conocimiento en enfermería debido a que han estado dedicadas a la enseñanza de otras enfermeras. Muchos aportes al saber científico profesional permanecen en un estado incipiente, debido en grail parte a que solamente un pequeño número de enfermeras le ha dedicado atención y energía para experimentar sus posibilidades. Las enfermeras han debido depender de lo que es reconocido y de lo que se ha obtenido de otras ocupaciones, en lugar de contar con programas activos de investigación para crear nuevos métodos de trabajo. Los conocimientos de enfermería sobre cómo aliviar el dolor, aumentar el confort del paciente, promover el descanso y el sueño, enseñar a los pacientes todo lo re. lativo a la solud y la enfermedad, asi como innumerables problemas de toda indole, permanecen a un nivel de subdesarrollo. Cuando en el programa de estudios se introducen nuevos conocimientos, se trata casí siempre de aportes de la medicina $u$ otras disciplinas paralelas, en lugar de ser el resultado de la reflexión y la experimentación de las propias enfermeras para mejorar el nivel de su profesión.

La escasez de personal de enfermería, la importante concentración de enfermeras en la enseñanza y la coordinación del trabajo de otras personas, así como la falta de desarrollo del cuerpo de conocimientos propios de la profesión, son los elementos que han contribuído a que el nivel de la atención al paciente $y$, por lo tanto, nas normas fijadas para los cuidados de la salud, sean inferiores a lo que sería deseable. Esta es la realidad tanto en los países industrializados como en los insuficientemente desarrollados, y es causa de frustración de enfermeras y de pacientes. Para complicar la situación actual, la demanda de la población por servicios de salud se ha acrecentado en muchos países, con lo cual la diferencia en. tre el tipo de cuidados de enfermería $y$ de salud deseado y el que se presta en la actualidad se hace cada día más evidente.

\section{CARACTERISTICAS DE UNA PROFESION}

El problema del nivel de los cuidados de enfermeria que se prestan es en parte producto de la autoestimación de la enfermeria misma como profesión. La opinión que las enfermeras tienen de sí mismas como profesionales no siempre coincide con la definición que otras personas adjudican a los profesionales. La mayoría de los sociólogos, por ejemplo, concuerdan en que hay tres criterios principales para definir una profesión. El primero es que los profesionales deben contar con un largo período de educación para recibir conocimientos especializados; el segundo, que una profesión está orietada a prestar un servicio o está preocupada de pres.. tar un servicio especial a gente que lo necesita. El tecer criterio, autonomía, se basa en los dos mencionados, educación y servicio. Autonomía profesional significa que son los miembros de una profesión quienes la reglamentain y la controlan. El argumento en favor de esta posición es que si el cuerpo de conocimientos de los profesionales $\epsilon$ en realidad tan especializado, nadie mejor que ellos mismos podrá definir los términos de un ejercício profesional seguro y efectivo. Una persona que no posee el conocimiento especial de una disciplina determinada no es is 
JACOX, A. - Un problema subestimado en enfermeria: la influencia que ejerce sobre la atención al paciente el bienestar económico y social de la enfermera. Rev. Bras. Enf.; DF, $32: 8-19,1579$.

más indicada para emitir juicios acerca de cómo esos conocimientos deben ser utilizados. Esta es la base sobre la que se asienta el concepto de que los profesionales deben tener la autoridad necesaria para controlar el ejercicio de su profesión.

Aunque la profesión produce las normas y las indicaciones acerca de cómo el trabajo debe ser realizado, no es común que un profesional supervise a otro profesional, salvo durante periodo de adiestramiento. Los profesionales se asesoran y consultan entre colegas, рето la responsabilidad de una decisión corresponde al profesional que la ha tomado. El juicio profesional significa le. utilización de los conocimientos especializados que se poseen, para tomar la mejor decisión de cómo aplicarlos a una situaciór determinada. En el mode!o profesional, el conocimientos es la basi que otorga autoridad a los miembros para tomar decisiones respecto al ejercicio de la profesión.

\section{CONFLICTOS EN LAS FUENTES DE AUTORIDAD}

Esto contrasta con la situación existente em organizaciones de gran complejidad dentro de las cuales prima la autoridad de cada cargo. Dichas organizaciones están generalmente estructuradas en base a una jerarquía de autoridad, o sea que en ellas cada persona deberá obedecer las órdenes impartidas por otra persona de jerarouía superior. Esto plantea un conflicto potencial cuando la organización emplea profesionales. La noción de que, por el hecho de desempeñar un cargo administrativo superior, cualquer persona está automáticamente autorizada a supervisar el trabajo de profesionales situados desde el punto de vista jerárquico en un nivel inferior, ha sido con- siderada como una situación en conflicto con el modelo profesional. En épocas anteriores los profesionales actuaban aisladamente o en pequeños grupos, en contacto directo con sus clientes. Hace relativamente poco tiempo que los profesionales son empleados eir gran número por organismos complejos. Los cambios producidos en las condiciones de empleo de los profesionales y en sus relaciones con los clientes, han sicio la causa de muchos problemas para determinar quién es la persona mejor calificada para tomar determinadas decisiones sobre servicios profesionales al público. El conflicto potencial entre la autorídad burocrática y la autoridad profesional es un problema que deben enfrentar los profesionales que trabajan en organizaciones.

De los criterios profesionales mencionados, y generalmente aceptados (largo período de aprendizaje, orientaciói y la prestación de servicios y autonomia), la enfermería ha dedicado principalmente su atención a los dos pr:meros habiéndose interesado menos en el de la autonomía. Las enfermeras, en general, se han interesado por elevar el nivel de su educación. Esto ha un esfuerzo internacional, que aún hoy persiste intensamente. Tampoco se pone en duda la orientación del ejercicio de su profesión; la enfermera presta un servicio que es cluaramente necesari's al público. Lo que si puede decirse es que no se ha prestado mayor atención a la dimensión de la autonomía de st? profesionalismo, a causa principalmente de que se espera obediencia de su parte. Los conceptos de obediencia ciega $\mathrm{y}$ autonomía son contradictorios. La atención que la educación y el servicio han merecido a la enfermera, y su desinterés por la autonomía profesional, han tenido consecuencias negativas para lid enfermería. 
JACOX, A. - Un problema subestimado en enfermeria: la influencia que ejerce sobre la atención al paciente el bienestar económico y social de la enfermera. Rev. Bras. Enf.; DF, $32: 8-19,1979$.

\section{EFECTOS NEGATIVOS SOBRE LA ENFERMERIA}

Uno de los efectos negativos de la falta de preocupación por la autonomía profesional es el hecho de que las enfermeras no han estado en condiciones de cotrolar el ejercicio de su propia profesión. Por el contrario, han debido llevar a cabo ordenes, incluso sobre asuntos de enfermeria, impartidas por superiores en la jerarquía administrativa, aún si los administradores fucra $\Omega$ o no enfermeras. Han debido también obedecer las órdenes de los médicus, aún cuando estas órdenes se relacionaban con los cuidados de enfermería más bien que la atención médica. Por supuesto, todas las personas que trabajan en una organización deben cumplir con una serie de reglamentos y disposiciones para que ésta funcione. Además, en la medida en que las enfermeras ejecutan funciones médicas delegadas, es apropiado que sean supervisadas por el y que cumplan sus órdenes. Pero en la medida en que ellas prestan cuidados de enfermería, no es apropiado pensar que médicos y otras personas que no sean enfermeras tengan los corocimientos o la autoridad para supervisar a las enfermeras. Es aquí que debe estabelecerse claramente la distinción entre la medicina y la enfermeria, con enfermeras controlando definidamente el ejercicio de su profesión.

A causa de su tradicional convicción de que debe estar superditada a otras personas, la enfermera, con frecuencia, no ha ocupado cargos que implicaran la toma de decisiones en el ámbito de la enfermería y menos aún en el de lia atención de salud en general. Aunque es hoy día cada vez más reconecido el hecho de que la atención de la salud comprende que la atención médica, la partjcipación de enfermeras en el proceso de toma de decisiones con respecto a atención de salud en general es extremadamente lenta en muchos países, y en otros ni siquiera existente. Coms las enfermeras no están en posiciones para la toma de decisiones sobre atención de salud, los presupuestos para los programas de atención de salud reflejan poca prioridad para enfermería.

Otro efecto de la falta de autonomía es la falta de iniciativa de las enfermeras en cuanto a luchar por obtener una remuneración justa y de acuerdo con su trabajo. En una sociedade donde la remuneración varía de acuerdo con el tipo de trabajo, los profesionales están en general mejor pagados que otros funcionarios con menor preparación. Pero esto no ha sucedido con la enfermería. Las enfermeras dedican varios años de su vida a recibir una instrucción especializada, y luego desempeñan duras y serias tareas de responsabilidad relativa al cuidado de los pacientes. A pesar de ello nunca han esperado ni se ha esperado que el salario que les corresponde sea de igual nivel que el de otros profesionales con similar preparación y responsabilidad.

Un estudio hecho en 1960 (1) sobre las condiciones de empleo de enfermeras en 50 países y territorios estableció que:

“...la remuneración por hora de las enfermeras de hospital es inferior a las tasas horarias del grupo peor pagado de trabajadores de la industria en casi la mitad de los países estudiados. En aproximadamente una tercera parte de los países, las jefas de enfermeras también perciben una remuneración por hora inferior a la del grupo peor pagado de trabajadores industriales, mientras que en las dos terceras partes de los paises, respecto de los que se dispone de datos sobre los dos grupos, las jefas de enfermeras cobran menos que el grupo de trabajadores calificados." 
JACOX, A. - Un problema subestimado en enfermeria: la influencia que ejerce sobre la atención al paciente el bienestar económico y social de la enfermera. Rev. Bras. Enf.; DF, $32: 8-19,1579$.

Aunque han transcurrido más de 16 años desde dicha encuesta, la situación no ha variado significativamente en muchos países. No solamente las enfermeras no son pagadas con el mismo criterio con que se paga a otros profesionales, sino que su salario ni siquiera es comparable con el de obreros noespecializados. Una vez más, ésto se debe en parte a que la mayoria de las enfermeras son mujeres y que el trabajo femenino es subestimado. Muchas enfermeras han aceptado este estado de cosas como algo normal, sin jamás decidirse a luchar por mejorar la situación.

Otro problema que a la vez deriva $y$ contribuye a la falta de control que la enfermera ejerce sobre su trabajo, es ei marco en el cual la enfermera recibe su educación. En casi todos los países la instrucción de profesionales está a cargo de las universidades. No ha sucedido asi con las enfermeras, quienes, generalmente, son educadas o adiestradas en hospitales. De esta manera están expuestas desde el principia de su instrucción a valores sólidamente establecidos en el sistema hospitalario, donde la autoridad para la toma de decisiones está en manos de médicos y administradores. La continuación de los programas de educación en ese tipo de institución contribuye a que la enfermera continúe creyendo que otros, que no pertenecen a la profesión tienen el derecho de controlar las decisiones relativas a los cuidados de enfermería.

Estos factores reflejan y consolidan la subestimación de la importancia de la enfermería que existe en demasiado lugares. Si las enfermeras han de mejorar la enfermería, deben estar preparadas para actuar de manera efectiva y responsable para cambiar la situación actual. Quienes poseen autoridad para tomar decisiones son generalmente reacios a delegarla en otros. Esto se aplica también a la enfermería, en que per- sonas que no son enfermeras controlan las decisiones profesionales que competen a las enfermeras.

\section{ASOCIACIONES PROFESIONALES Y ACCIÓN COLECTIVA}

Los profesionales defienden sus intereses de varias maneras: una de ellas es la formación de asociaciones profesionales. La asociación profesional está basada en el principia de la acción colectiva, o sea, que las personas alcanzan más fácilmente sus metas cuando actúan como grupo que cuando lo hacen separadamente como individuos. Las asociaciones profesionales tienen la doble función de mantener una alta calidad de servicio para sus clientes y de proteger los intereses económicos y sociales de sus miembros. Algunas de las actividades de una asociación profesional son dirigidas hacia una de éstas funciones principales, otras hacia la otra, y algunas hacia ambas. A veces sucede que ciertas actividades relativas a una de las funciones entran en conflicto con la otra. Corresponde a los miembros de la asociación mantener un interés apropiado y un equilibrio equitativo entre protección del público y la de los profesionales. La habilidad de una profesión para lograr sus objetivos depende en bueno parte de la fuerza colectiva de sus miembros expresada por medio de las acciones de su asociación profesional. La forma que adopta una asociación profesional y las relaciones que estableca con otros grupos para lograr sus objetivos dependerá de la sociedade en que se desenvuelva, de ahí la variedad de asociaciones de enfermeras que existen. La forma más común que se presenta es la de una asociación compuesta solamente de miembros de una misma profesión.

En unos pocos países sin embargo, aparecen representadas en una asocia- 
JACOX, A. - Un problema subestimado en enfermeria: la influencia que ejerce sobre la atención al paciente el bienestar económico y social de la enfermera. Rev. Bras. Enf.; DF, $32: 8-19,1979$.

ción arias profesiones y ócupaciones distintas pero con intereses comunes. En éstos casos los miembros de una profesión generalmente, forman una subdivisión de la asociación. En casi todos los países, la relación entre la asociación y los organismos gubernamentales es independiente, y es la asociación quien debe hacer las gestiones con los funcionarios gubernamentales (en las ramas legislativa y administrativa), para influir en un sentido determinado sobre las decisiones que se adopten en materia de enfermeria y salud en general. En otros países las asociaciones desempeñan un papel diferente con respecto al gobierno, como por ejemplo una asociación de enfermeras que opera esencialmente como parte integrante del gobierno y se ha fijado como meta "una cooperación constante con órganos administrativos del Estado para asegurar a la comunidad una atención de enfermeria adecuada". Cualquiera sea la forma adoptada por la asociación y su relación con otros grupos, es básico el principio que son los profesionales quienes mantienen el control de las decisiones relativas al ejercicio de su profesión.

Los métodos usados por los profesionales para lograr sus objetivos varian con las condiciones sociales y culturales y con las circunstancias cambiantes. A algunos de éstos métodos ya nos hemos referido anteriormente. Uno de ellos es tratar de obtener la aprobación de una legislación favorable al ejercicio de la profesión y al bienestar económico y social de sus miembros. Otro consiste en establecer contactos y relaciones de trabajo con integrantes del gobierno para que las decisiones que afecten el ejercicio de una profesión sean precedidas del mayor aporte de datos posible por parte de sus miembros. Un tercer método de acción es el desarrollo de normas para el ejercicio, la enseñanza, da autorización para ejercer y para otras areas similares de interés profesional. Establecer tales normas es una acción aprobada por las enfermeras desde hace ya mucho tiempo y en la que muchas tienen gran experiencia.

Los profesionales también se ocupan de negociaciones colectivas de trabajo. "La negociación colectiva es un proceso de adopción de decisiones. Su propósito primordial es llegar a convenir en un conjunto de normas que rijan las cuestiones de fondo y de procedimiento de la relación de empleo, al igual que las relaciones entre las propias partes en la negociación." (2)

Existen diferentes formas de negociaciones colectivas, la más usual es la qua. tiene como resultado un contrato, generalmente escrito, entre un grupo de empleados y un empleador. Otras formas de negociación son las discusiones que tienen lugar en el seno de organismos centrales bipartidos, como ia Conferencia Nacional de Trabajo en Paquistán, y las discuciones de un gobierno con empleadores y sindicatos acerca de, por ejemplo, una nueva ley laboral. “...existe negociación colectiva siempre que se entable una discusión entre empleadores y empleados, y posiblemente el gobierno, sobre problemas económicos y sociales, durante la cual las diversas partes tratan de llegar a un compromiso por via de concesiones reciprocas." (3)

En el tipo de negociaciones colectivas en que un grupo de empleados discute salario, condiciones de trabajo y otros asuntos, con un empleador determinado, los empleados eligen generalmente varios de sus miembros para que los representen en las discusiones con los representantes del empleador. La forma de gobierno y las leyes que rigen la negociación ejercen una influencia determinada sobre quienes pueden representar a los empleados, el procedi- 
JACOX, A. - Un problema subestimado en enfermeria: la influencia que ejerce sobre la atención al paciente el bienestar económico y social de la enfermera. Rev. Bras. Enf.; DF, $32: 8-19,1579$.

miento seguido durante las discusiones, y los puntos que podrán ser discutidos. Dentro de este contexto jurídico y so. cial, los problemas relativos a las condiciones de trabajo y las relaciones laborales son solucionados mediante la negociación y el compromiso entre las partes concernidas. En algunos países están prohibidas las negociaciones colectivas; en otros se garantiza a tod.) empleado el derecho a participar en negociaciones colectivas si así lo desea. En países en que el empleo es o público o privado, las leyes y reglamentos distinguen a menudo la conducta a seguir en negociaciones en uno y otro sector.

Si bien las negociaciones colectivas han sido tradicionalmente utilizadas por obreros (especializados o no), hoy día el procedimiento es empleado también y cada día más a menudo por profesionales asalariados. Su uso por profesionales es el resultado de la tendencia actual de un mayor empleo de profesionales en organizaciones diversas. En consecuencia, los profesionales se encuentran en una posición salarial semejante a la de otros empleados. Los profesionales ya no son pagados directamente por el cliente sino que por la organización, que a su vez cobra sus honorarios al cliente o a terceras partes, como compañías ds seguros y organismos gubernamentales, por ejemplo Además del ingreso a organizaciones de profesionales anteriormente independientes, los miembros de algunas ocupaciones ya empleadas por organizaciones han tratado de convertilas en profesiones. Este es el caso de enfermeras, maestros, trabajadores sociales, y otros. A medida aue más profesionales pasan a recibir salarios fijos, la negociación colectiva gana en aceptación entre ellos.

De acuerdo con que los empleados sean o no profesionales, la elección del representante en la negociación se vuel. ve muy importante. Generalmente esa elección queda a cargo de los empleados mismos. En las negociaciones en que una de las partes está compuesta de enfermeras, es generalmente preferible que sea la asociación de enfermeras profesionales quien asuma este papel. En algunos países con organizaciones que representan una gran variedad de empleados, incluyendo a las enfermeras, la legislación especifica que son éstas las organizaciones encargadas de negociar para las enfermeras. Aún en éstos casos las enfermeras sin embargo, deberán tratar de retener una mayor influencia sobre las decisiones relativias a sus condiciones de empleo para asegurar que esas decisiones contribuyan de la mejor manera posible a un ejercicio profesional seguro y eficaz.

Es importante que todo grupo de empleados que intente negociar colectivamente conozca plenamente las disposiciones jurídicas existentes en el país relativas a negociaciones. Información más detallada sobre los aspectos técni$\cos$ y jurídicos de la negociación colectiva en muchos países se encuentra en los libros incluídos en la bibliografia anotada al final de este trabajo. Las personas interesadas deberán consultar trabajos que se refieran a la situación específica en sus países respectivos. Puede obtenerse más información a] respecto en la Oficina Internacional del Trabajo en Ginebra, Suiza.

En general la negociación colectiva se ha utilizado más que nada para mejorar salarios y condiciones de trabajo, tales como seguridad social, horarios, vacaciones, etc. Cuando intervienen profesionales en las negociaciones colectivas, éstas sirven a menudo para influenciar directamente aspectos del ejercicio de la profesión, así como para mejorar sus condiciones económicas. Los profesores, por ejemplo, han negociado la organización de conferencias de educación, 
JACOX, A. - Un problema subestimado en enfermeria: la influencia que ejerce sobre la atención al paciente el bienestar económico y social de la enfermera. Rev. Bras. Enf.; DF, $32: 8-19,1979$.

número de alumnos asignado a cada profesor, y la seguridad de que su tiempo no se ocupe en tareas que podrían ser llevadas a cabo más eficazmente por personas con menor preparación. Los médicos han negociado el número de profesionales que debian ser asignados a un serviço específico y a un número determinado de pacientes, así como mejoras en el equipo y material de que disponen para realizar su trabajo.

Las enfermeras han utilizado la negociación colectiva para lograr una serie de objetivos, tales como mejora de salarios y condiciones de trabajo, y medidas para mejorar la atención al paciente. Por ejemplo, un grupo de enfermeras en un hospital negoció hasta que el empleador accedió, que ninguna enfermera fuera asignada a unidades de tratamiento intensivo altamente especializado, sin haber pasado antes por un curso adecuado de especialización para trabajar en este tipo de unidades. Otro grupo rehusó la firma de un acuerdo con el empleador hasta que la limpieza y tareas similares que distraían tiempo de las enfermeras para la atención de los pacientes, fueron asignadas a otros grupos de trabajadores. En muchos lugares las enfermeras han negociado acuerdos según los cuales se han foramdo comités de atención de pacientes, donde las enfermeras desempeñan un papel activo en el establecimiento de procedimientos seguros y eficaces. Generalmente todas estas conquistas y muchas otras no hubieran sido posibles si las enfermeras hubieran actuado aisladamente: pero cuando ellas se han unido y han accedido a un poder colectivo y a la obtención de metas comunes, las enfermeras han podido lograr mucho para mejorar la atención de enfermería.

Resumiendo, los profesionales actúan colectivamente para mejorar el ejercicio de su profesión y sus condiciones de empleo, y para ello varios tipos de acción son posibles de acuerdo al país y a las circunstancias de cada caso. El principio de la acción colectiva es fundamental para lograr objetivos profesionales.

\section{RESISTENCIA DE LAS ENFERMERAS} A UTILIZAR LA ACCION COLECTIVA

Aún cuando el principio de acción colectiva ha sido desde hace tiempo aceptado y puesto en práctica por grupos profesionales, muchas enfermeras son renuentes a participar en tales actividades. Para ello existe un buen número de razones, la más importante de las cuales es quizá el hecho anteriormente citado, de la importancia atribuída a los factores obediencia y otro tipo de comportamiento considerado "apropiado" para las mujeres. Es personalmente dificil para alguien a quien se ha enseñado a ser pasivo y no agresivo, decidirse a intervenir en la acción colectiva. Esta resistencia inconsciente es reforzada por las criticas de que son objeto las enfermeras y otras mujeres que defienden sus intereses y aquellos de su trabajo. Un problema relacionado con éste, es el de la falta de experiencia de muchas enfermeras en lo que se refiere a la acción colectiva. En muchos países las mujeres no intervienen aún hoy en actividades politicas ni participan en la toma de decisiones fuera del hogar, y a veces ni siquiera en el hogar. Cuando ocupan cargos administrativos es, generalmente, para supervisar el trabajo de otras mujeres, raramente a hombres y mujeres. Finalmente, las mujeres profesionales, en particular, no tienen experiencia en la utilización de técnicas de negociaciones colectivas. A pesar de todos estos factores, es importante que las enfermeras lleguen a reconocer la necesidad de tomar posición y decidirse a actuar en el interés de la enfermería y las enfermeras. 
JACOX, A. - Un problema subestimado en enfermeria: la influencia que ejerce sobre la atención al paciente el bienestar económico y social de la enfermera. Rev. Bras. Enf.; DF, $32: 8-19,1579$.

\section{EL IMPERATIVO DE LA ACCIÓN COLECTIVA DE PARTE DE LAS ENFERMERAS}

Una de las consecuencias más desafortunadas de la identificación de un tipo de trabajo como "trabajo de mujeres", es que el trabajo mismo se devalúa. Este es el caso en la enfermería, puesto que es la profesión que posee el porcentaje más alto de mujeres que ninguna otra. Las enfermeras deben revalrizarse a si mismas y deben revalorizar el trabajo que desempeñan, pues si no lo hace ellas mismas, nadie lo hará. Si las enfermeras están auténticamente interesadas en mejorar el ejercicio de la enfermería y con ello mejorar la atención de la salud en general, deben actuar en todo sentido de la palabra como profesionales, sin tratar de evitar el riesgo de, al perseguir el logro de los objetivos propuestos, adoptar posiciones con las que no todos estarán de acuerdo. Cuando una acción agresiva en favor del mejoramiento de la enfermería es catalogada por personas que no son enfermeras como "no-profesional", no debe verse en ello algo más de lo que realmente significa-un intento más por prolongar el control de las enfermeras y de la enfermería. La noción de que una acción decidida por parte de enfermeras actuando individual- y colectivamente, es antiprofesional, debe ser firmemente resistida. Si el resultado de esa acción es el mejoramiento del ejercicio de la profesión, será por el contrario, un comportamiento profesional del más alto nivel.

Algunas enfermeras pueden no estar interesadas en recibir mejores salarios, obtener condiciones de trabajo más favorables, o ejercer un mayor control de su profesión; ellas tienen el derecho, por supuesto, de no sentirse preocupadas por su propio bienestar. Es impor-tante sin embargo, considerar las con- secuencias que a largo plazo esta actitud de una mayoria de enfermeras que piensen de esta manera y actúen consecuentemente, significará para la enfermería. Si la enfermería continúa siendo considerada como una ocupación en la que se encontrará arduo trabajo. muchas frustraciones debido a la im.posibilidad de ejercer de acuerdo con normas profesionales, bajos salarios $y$ condiciones inadecuadas, seguirá siendo dificil reclutar un número suficiente de personas inteligentes y sólidamente preparadas, motivadas para permanecer dentro de la profesión y mejorarla. Enfermeria debe competir con otras profesiones que también hacen una contri-bución social, para atraer candidatos calificados. Pero no puede hacerlo coil exito si continúa en situación de desventaja con respecto a la satisfación en el trabajo y a la condición económica de sus miembros. Es importante que una mayoría de enfermeras se decida a hacer de la enfermería una profesión atractiva, no sólo por la importante contribución social que puede llegar a significar, sino también por convertirla en una experiencia positiva y satisfactoria, adecuadamente remunerada, a la par de otras profesiones. La mejora de los salarios y de las condiciones de trabajo pueden no ser importantes para alguna enfermera" o grupos de enfermeras determinadas, pero es un elemento fundamental para el mejoramiento de la enfermería en general.

Los servicios que las enfermeras desempeñan son de importacia vital. La promoción y la restauración de la salud, la prevención de la enfermedad $y$ la tención de los enfermos son funciones esenciales en toda sociedad. Si las enfermeras quieren asegurar a la sociedad el nivel más elevado posible de servicios de enfermeria y si quieren además trabajar eficazmente para obte ner un mejoramiento de la atención de 
JACOX, A. - Un problema subestimado en enfermeria: la influencia que ejerce sobre la atención al paciente el bienestar económico y social de la enfermera. Rev. Bras. Enf.; DF, $32: 8-19,1579$.

salud, deben aceptar participar en las actividades tendientes al logro de esos objetivos. Deben reconocer clarafente la relación que existe entre condiciones de empleo de los profesionales y la calidad del servicio que el cliente recibe de ellos. Solamente cuando una mayoría de en- fermeras comprenda ésto y actúe co. lectiva- y positivamente para cambiar de manera drástica las condiciones existentes, será posible hacer realidad y poner en práctica los ideales que la profesión ha mantenido desde hace tanto tiempo.

\section{REFERENCIAS}

1. Oficina Internacional del Trabajo: Empleo y condiciones de trabajo de las enfermeras, pág. 111 (Ofícina Internacional del Trabajo, Ginebra, 1960).

2. Oficina Internacional del Trabajo: La negociación colectiva en países industrializados con economia de mercado. Estudios y documentos, nueva serie, núm. 80, pág. 7 (Oficina Internacional del Trabajo, Ginebra, 1974).

3. Oficina Internacional del Trabajo: Organización Internacional del Trabajo, "Background paper prepared by the ILO. Tripartite Advisory Meeting on Collective Bargaining", pág. 3 (Ginebra, 10-19 mayo, 1976).

\section{BIBLIOGRAFIA}

Alexandre, $R . J .:$ The Labour Movement in Brazil, Argentina and Chile (McGrawHill, New York, 1962).

Brown, E. C.: Soviet Trade Unions ans] Labour Relations (Harvard University Press, Cambridge, 1966).

"Collective Action and Control of Practice by Professionals", Nursing Forum (Vol. 10, 1971).

Oficina Internacional del Trabajo: Las negociaciones colectivas: Manual de educación obrera (Oficina Internacional de? Trabajo, Ginebra, 1969).

Oficina Internacional del Trabajo: La negociación colectiva en países industrializados con economía de mercado, Estudios y documentos, nueva serie, núm. 80 (Oficina Internacional del Trabajo, Ginebra, 1974).

Cook, A. H.: Japanese Trade Unionism (Cornell University Press, Ithaca, 1966).

Galenson, W. (ed.): Labour and Economic Development (John Wiley and Sons, New York, 1959).

Galenson, $W$. (ed.): Labour in Developing Countries (University of Colifornia Press, Berkeley and Los Angeles, 1962).

Gladstone, A. y Ozaki, M.: Reconocimiento de los sindicatos y negociación colectiva, Revista Internacional del Trabajo (agosto-septiembre de 1975).

"How Collective Bargaining Works", Nursing 76, pp. 77-85 (June 1976).
Oficino Internacional del Trabajo: "Background paper prepared by the ILO. Tripartite Advisory Meeting on Collective Bargaining", (Ginebra, 10-19 mayo, 1976).

Isaac, J. S. y Ford, G. W. (eds.) : Australian Labour Relations Readings (Sun Brooks, Melbourne, 1966).

Lorwin, $\boldsymbol{V}$. R.: The French Labour Movement (Harvard University Press, Cambridge, 1955).

Myers, C. A.: Labour Problems in the Industrialization of India (Harvard University Press, Cambridge, 1953).

Roberts, B. C. y De Bellecombe, L. G.: Collective Bargaining in African Countries (St. Martin's Press, New York, 1967).

Sturmthal, A. F. (ed.): Contemporary Collective Bargaining in Seven Countries (Cornell University Press, Ithaca, 1957).

Walker, $K .:$ Industrial Relations in Australia (Harvard University Press, Cambridge, 1956).

Consultar también:

"Economic and General Welfare Department Bibliography", American Nurses Association, 2420 Pershing Road, Kansas City, Missouri 64103, USA (December 1975). 